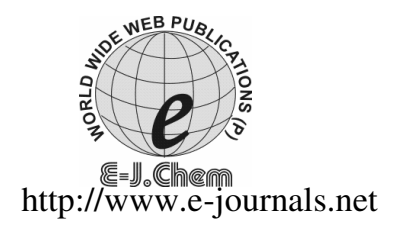

\title{
Synthesis and Pharmacological Evaluation of Some Phenylpyrazolo Indoquinoxaline Derivatives
}

\author{
CH.SRIDEVI ${ }^{*}$, K.BALAJI and A.NAIDU \\ *Dept. of Pharmaceutical Chemistry, Geethanjali College of Pharmacy \\ Cheeryal(V),Keesara(M), Hyderabad.501301, India \\ sridevi.phd@gmail.com.
}

Received 1 July 2010; Revised 1 November 2010; Accepted 6 November 2010

\begin{abstract}
Indoloquinoxalin was fused with 2,3 diphenyl quinoxaline by a methylene bridge which was then allowed for acetylation. The acetylated product was made to react with different aromatic aldehydes to give chalcones. Chalcones refluxed with substituted acid hydrazides to afford different indoloquinoxaline pyrazolines. The structure of chalcones and indoloquinoxaline pyrazolines were confirmed by M.P, TLC and spectral data. All the synthesized compounds were screened for their antioxidant, antiinflammatory and antihistamic activities.
\end{abstract}

Keywords: Indoloquinoxalin, Pyrazoline, Antioxidant, Anti-inflammatory and Antihistaminic activity.

\section{Introduction}

Quinoxaline derivatives have been reported to possess a wide variety of biological activities ${ }^{1-3}$. Notable among these are antioxidant, anti-inflammatory antimicrobial, anticancer and antihistamic activities. Drugs having pyrazoline ring system ${ }^{4-6}$ are well known for their anti-inflammatory, antioxidant, antihistamic, antimicrobial, antidepressant, hypoglycemic, hypotensive, anticarcinogenic activities etc. In view of the above facts, we report here in the synthesis of some indoloquinaxoline pyrazoline derivatives by condensing indoloquinoxaline chalcones with different aromatic acid hydrazides. All the synthesized compounds (IQVI 1- IQVI 15) were screened for their antioxidant, anti-inflammatory and antihistamic activities. The structure of chalcones and indoloqunoxaline pyrazolines were confirmed by M.P, TLC, Spectral data (Table $1 \& 2$ ).

\section{Experimental}

The melting point of the compounds was determined on a Thoshniwal electric melting point apparatus and the values were uncorrected. I.R spectra of the compounds were recorded on a Thermo Nicolet Nexus 670-FTIR, IICT, Hyderabad using $\mathrm{KBr}$ disc method. ${ }^{1} \mathrm{H}$ NMR spectra were recorded on Avance-300, IICT, Hyderabad using $\mathrm{CDCl}_{3}$ as solvent. Mass spectra were recorded on GCMS-Auto tuning-EI at IICT, Hyderabad. All the solvents used were of analytical grade. 
Table 1. Physical data of phenylpyrazolo indoloquinoxaline derivatives

\begin{tabular}{ccccccc}
\hline Comp. & \multirow{2}{*}{$\mathrm{X}$} & $\mathrm{Ar}$ & $\begin{array}{c}\text { Molecular } \\
\text { Formula }\end{array}$ & $\begin{array}{c}\text { Melting point } \\
\text { range, }{ }^{\circ} \mathrm{C}\end{array}$ & $\begin{array}{c}\% \\
\text { Yield }\end{array}$ & $\begin{array}{c}\mathrm{R}_{\mathrm{f}} \\
\text { value }\end{array}$ \\
\hline IQVI1 & $\mathrm{H}$ & $\mathrm{C}_{6} \mathrm{H}_{5}$ & $\mathrm{C}_{52} \mathrm{H}_{37} \mathrm{~N}_{7} \mathrm{O}$ & $119-120$ & 62 & 0.60 \\
IQVI2 & $\mathrm{OH}$ & $\mathrm{C}_{6} \mathrm{H}_{5}$ & $\mathrm{C}_{52} \mathrm{H}_{37} \mathrm{~N}_{7} \mathrm{O}_{2}$ & $122-123$ & 45 & 0.9 \\
IQVI3 & $\mathrm{F}$ & $\mathrm{C}_{6} \mathrm{H}_{5}$ & $\mathrm{C}_{52} \mathrm{H}_{36} \mathrm{~N}_{7} \mathrm{O}_{3} \mathrm{~F}$ & $158-160$ & 75 & 0.66 \\
IQVI4 & $\mathrm{Cl}$ & $\mathrm{C}_{6} \mathrm{H}_{5}$ & $\mathrm{C}_{52} \mathrm{H}_{36} \mathrm{~N}_{7} \mathrm{OCl}$ & $165-166$ & 78 & 0.7 \\
IQVI5 & $\mathrm{OCH}_{3}$ & $\mathrm{C}_{6} \mathrm{H}_{5}$ & $\mathrm{C}_{53} \mathrm{H}_{39} \mathrm{~N}_{7} \mathrm{O}_{2}$ & $123-125$ & 63 & 0.82 \\
IQVI6 & $\mathrm{H}$ & $\mathrm{OHC}_{6} \mathrm{H}_{4}$ & $\mathrm{C}_{52} \mathrm{H}_{37} \mathrm{~N}_{7} \mathrm{O}_{2}$ & $119-120$ & 32 & 0.88 \\
IQVI7 & $\mathrm{OH}$ & $\mathrm{OHC}_{6} \mathrm{H}_{4}$ & $\mathrm{C}_{52} \mathrm{H}_{37} \mathrm{~N}_{7} \mathrm{O}_{3}$ & $122-123$ & 57 & 0.87 \\
IQVI8 & $\mathrm{F}$ & $\mathrm{OHC}_{6} \mathrm{H}_{4}$ & $\mathrm{C}_{52} \mathrm{H}_{36} \mathrm{~N}_{7} \mathrm{O}_{2} \mathrm{~F}$ & $158-160$ & 67 & 0.8 \\
IQVI9 & $\mathrm{Cl}$ & $\mathrm{OHC}_{6} \mathrm{H}_{4}$ & $\mathrm{C}_{52} \mathrm{H}_{36} \mathrm{~N}_{7} \mathrm{O}_{2} \mathrm{Cl}$ & $165-166$ & 68 & 0.89 \\
IQVI10 & $\mathrm{OCH}$ & $\mathrm{OHC}_{6} \mathrm{H}_{4}$ & $\mathrm{C}_{53} \mathrm{H}_{39} \mathrm{~N}_{7} \mathrm{O}_{3}$ & $112-115$ & 54 & 0.83 \\
IQVI11 & $\mathrm{H}$ & $\mathrm{ClC}_{6} \mathrm{H}_{4}$ & $\mathrm{C}_{52} \mathrm{H}_{36} \mathrm{~N}_{7} \mathrm{OCl}$ & $119-120$ & 58 & 0.78 \\
IQVI12 & $\mathrm{OH}$ & $\mathrm{ClC}_{6} \mathrm{H}_{4}$ & $\mathrm{C}_{52} \mathrm{H}_{36} \mathrm{~N}_{7} \mathrm{O}_{2} \mathrm{Cl}$ & $120-123$ & 72 & 0.85 \\
IQVI13 & $\mathrm{F}$ & $\mathrm{ClC}_{6} \mathrm{H}_{4}$ & $\mathrm{C}_{52} \mathrm{H}_{35} \mathrm{~N}_{7} \mathrm{OCF}$ & $158-160$ & 76 & 0.9 \\
IQVI14 & $\mathrm{Cl}$ & $\mathrm{ClC}_{6} \mathrm{H}_{4}$ & $\mathrm{C}_{52} \mathrm{H}_{35} \mathrm{~N}_{7} \mathrm{OCl}$ & $160-163$ & 66 & 0.89 \\
IQVI15 & $\mathrm{OCH} \mathrm{O}_{3}$ & $\mathrm{ClC}_{6} \mathrm{H}_{4}$ & $\mathrm{C}_{53} \mathrm{H}_{38} \mathrm{~N}_{7} \mathrm{O}_{2} \mathrm{Cl}$ & $120-123$ & 62 & 0.75 \\
\hline
\end{tabular}

Table 2. Spectral data of phenyl pyrazolo indoloquinoxaline derivatives

\begin{tabular}{cccc}
\hline Compd & $\mathrm{M}^{+1}$ & ${ }^{1} \mathrm{H} N \mathrm{~N} R, \mathrm{ppm}$ & $\mathrm{IR}, \mathrm{cm}^{-1}$ \\
\hline IQVI1 & $775,776,777$ & $7.17,7.48-7.95,7.32,7.48,3.8,3.9,4.9$ & $3030,2945,1675$ \\
IQVI2 & $791,792,793$ & $7.17,7.48-7.9,7.32-7.48,3.8,3.9,4.8,5.0$ & $3350,3030,2945,1675$ \\
IQVI3 & $793,794,795,796$ & $7.16,7.48-7.92,7.32-7.48,3.8,3.92$, & $3010,2985,1685$ \\
& & $4.8,6.92$ & \\
IQVI4 & $809,810,811,812$ & $7.16,7.48-7.92,7.32-$ & $3027,2955,1695$ \\
& & $7.48,3.8,3.92,4.7,7.06-7.22$ & \\
IQVI5 & $805,806,807,808$ & $7.16,7.48-7.92,7.32-7.48$, & $3010,2975,1663$ \\
& & $3.8,3.9,3.73,6.72$ \\
IQVI6 & $791,792,793,794$ & $7.15,7.48-7.92,7.32-7.48$, & $3033,2955,1645$ \\
& & $3.8,3.92,4.8,5.1$ & \\
IQVI7 & $807,808,809,810$ & $7.16,7.4-7.9,7.32-7.48,3.8,3.92,4.8$, & $3382,3037,2949,1677$ \\
& & $6.68-7.78,5.0$ & \\
IQVI8 & $809,810,811,812$ & $7.17,7.48-7.92,7.32-7.48$, & $3387,3036,2945,1675$ \\
& & $3.82,3.92,4.8,6.14-7.7,6.98-7.1,5.2$ & \\
IQVI9 & $825,826,827,828$ & $7.16,7.48-7.9,7.32-7.4$, & $3389,3024,2945,1675$ \\
& & $3.8,3.92,4.8,6.91-7.78,5.0$ & \\
IQVI10 & $821,822,823,824$ & $7.16,7.48-7.92,7.32-7.48$, & $3387,3030,2945,1675$ \\
& & $3.8,3.92,4.8,6.72-7.78,3.73,5.0$ & \\
IQVI11 & $809,810,811,812$ & $7.17,7.48-7.92,7.32-7.48$, & $3028,2925,1678$ \\
IQVI12 & $825,826,827,828$ & $7.16,7.48-7.92,7.32-7.48$, & $3037,2948,1679$ \\
IQVI13 & $827,828,829,830$ & $7.15,7.4-7.92,7.32-7.48$, & $3072,2946,1677$ \\
IQVI14 & $843,844,845,846$ & $7.16,7.48-7.92,7.32-7.48$, & $3014,2955,1695$ \\
IQVI15 & $839,840,841,842$ & $7.16,7.48-7.92,7.32-7.48$, & $3039,2935,1676$ \\
\hline
\end{tabular}




\section{Preparation}

Synthesis of 2-((2,3-diphenylquinaxaline-6-yl)methyl)-6H-indolo[2,3-b] quinoxaline ${ }^{7}$

Equimolar quantities of $6 H$-indolo[2,3-b]quinoxalin (IQ I) and 2,3-diphenyl quinoxaline (IQ II) taken in a suitable solvent with 35 parts formaldehyde solution and $35 \% \mathrm{HCl}$, The solution was stirred for $4 \mathrm{~h}$ at $70{ }^{\circ} \mathrm{C}$ using magnetic stirrer. The purity of the compound was checked by TLC and melting point.

General procedure for Synthesis of 1-(2-((2, 3-diphenylquinoxaline-6-yl) methyl)$6 H$-indolo [2, 3-b] quinoxaline-6-yl) propan-2one ${ }^{8}$

2-((2, 3-Diphenylquinaxaline-6-yl)methyl)-6H-indolo[2, 3-b]quinoxaline (IQ III) (0.01 M) and chloro acetone $(0.01 \mathrm{M})$ were taken into $250 \mathrm{~mL}$ round bottom flask. $150 \mathrm{~mL}$ of dry acetone and $30 \mathrm{~g}$ of anhydrous potassium carbonate was added to it and the reaction mixture was refluxed for $6 \mathrm{~h}$ at $75{ }^{\circ} \mathrm{C}$. Filtrate obtained was concentrated under vacuum. The purity of the compound (IQ IV) was checked by TLC and melting point.

General procedure for Synthesis of 1-(2-((2, 3-diphenylquinaxaline-6-yl) methyl)6H-indolo[2, 3-b]quinaxaline-6-yl)but-3-en-2-one ${ }^{9}$

A solution of $\mathrm{NaOH} / \mathrm{KOH}(8 \mathrm{~mL}, 10 \%$ in water) was added drop wise to a well stirred solution of (0.01 M) 1-(2-((2, 3-diphenylquinaxaline-6-yl) methyl)-6H-indolo[2, 3-b] quinaxaline-6-yl) propan-2one (IQ IV) and $0.01 \mathrm{M}$ appropriate $p$-aryl aldehyde in $20 \mathrm{~mL}$ ethanol at cold temperature. The reaction mixture was stirred for $24 \mathrm{~h}$. at room temperature. Then diluted with ice water and acidified with Con.HCl. The product was filtered and recrystallized with aqueous ethanol. The purity of the compounds (IQV 1-IQV 5) was checked by TLC and melting point.

General procedure for Synthesis of 4,5-dihydro-5-phenyl-3-((2-((2,3diphenylquinaxaline-6-yl)methyl)-6H-indolo[2,3-b]quinaxaline-6-yl)pyrazol-1$y l)\left(\right.$ phenyl)methanone $e^{10}$

1-(2-((2, 3-Diphenylquinaxaline-6-yl) methyl)-6H-indolo[2, 3-b]quinaxaline-6-yl)but-3-en2-one (i.e., Chalcones) $(0.01 \mathrm{M})$ and aromatic acid hydrazide $(0.02 \mathrm{M})$ were taken in $20 \mathrm{~mL}$ glacial acetic acid and refluxed for $10 \mathrm{~h}$ above $130{ }^{\circ} \mathrm{C}$. The reaction mixture was concentrated and poured in $300 \mathrm{~mL}$ of ice-cold water and recrystallized with aq.ethanol. The purity of the compounds (IQVI 1- IQVI 15) was checked by TLC and melting point. The physical data are shown in Table 1.

\section{Pharmacological evaluation}

Antioxidant activity ${ }^{11}$

DPPH method

All drugs have been diluted in $95 \%$ ethanol to get $1000 \mu \mathrm{g}, 500 \mu \mathrm{g}, 250 \mu \mathrm{g}, 100 \mu \mathrm{g}, 50 \mu \mathrm{g}$ and $25 \mu \mathrm{g}, 10 \mu \mathrm{g} / \mathrm{mL}$ concentrations. DPPH solution $(2 \mu . \mathrm{mol})$ has been prepared by $95 \%$ ethanol. Then $0.5 \mathrm{~mL}$ of drug solution and $0.5 \mathrm{~mL}$ of DPPH solution (freshly prepared) were added. $0.5 \mathrm{~mL}$ of DPPH solution and $0.5 \mathrm{~mL}$ of ethanol were used as control. Reaction mixture was allowed for $20 \mathrm{~min}$. UV absorbance was measured at $517 \mathrm{~nm}$. The percentage of scavenging has been calculated by the equation given below. Ascorbic acid was used as standard drug (Table 3). 
Table 3. Antioxidant activity of phenylpyrazolo indoloquinoxaline derivatives

\begin{tabular}{|c|c|c|c|c|c|c|c|c|}
\hline Compd & Control & $10 \mu \mathrm{g}$ & $25 \mu \mathrm{g}$ & $50 \mu \mathrm{g}$ & $100 \mu \mathrm{g}$ & $250 \mu \mathrm{g}$ & $500 \mu \mathrm{g}$ & $1000 \mu \mathrm{g}$ \\
\hline STD & $\begin{array}{c}0.1 \pm \\
0.092\end{array}$ & $\begin{array}{c}0.06+ \\
0.017 \\
(40)\end{array}$ & $\begin{array}{c}0.029+ \\
0.016 \\
(71)\end{array}$ & $\begin{array}{c}0.020+ \\
0.0141 \\
(80)\end{array}$ & $\begin{array}{c}0.018 \pm \\
0.01 \\
(82)\end{array}$ & $\begin{array}{c}0.012+ \\
0.009 \\
(88)\end{array}$ & $\begin{array}{c}0.010 \pm \\
0.008 \\
(90)\end{array}$ & $\begin{array}{c}0.005 \pm \\
0.007 \\
(95)\end{array}$ \\
\hline IQVI 1 & $\begin{array}{l}0.12+ \\
0.092\end{array}$ & $\begin{array}{c}0.11 \pm \\
0.07 \\
(8.33)\end{array}$ & $\begin{array}{l}0.1 \pm \\
0.06 \\
(16.66)\end{array}$ & $\begin{array}{c}0.091 \\
0.057 \\
(24.16)\end{array}$ & $\begin{array}{c}0.09 \pm \\
0.049 \\
(25)\end{array}$ & $\begin{array}{c}0.088 \pm \\
0.02 \\
(26.66)\end{array}$ & $\begin{array}{c}0.08+ \\
0.013 \\
(33.33)\end{array}$ & $\begin{array}{c}0.07 \pm \\
0.001 \\
(41.66)\end{array}$ \\
\hline IQVI 2 & $\begin{array}{c}0.100 \pm \\
0.092\end{array}$ & $\begin{array}{c}0.089 \pm \\
0.079 \\
(11.0)\end{array}$ & $\begin{array}{c}0.083 \pm \\
0.074 \\
(17.0)\end{array}$ & $\begin{array}{c}0.075 \pm \\
0.071 \\
(25.0)\end{array}$ & $\begin{array}{c}0.059 \pm \\
0.05 \\
(41.0)\end{array}$ & $\begin{array}{c}0.059 \pm \\
0.05 \\
(41.0)\end{array}$ & $\begin{array}{c}0.05 \pm \\
0.03 \\
(49.0)\end{array}$ & $\begin{array}{c}0.042 \pm \\
0.012 \\
(58.0)\end{array}$ \\
\hline IQVI 3 & $\begin{array}{l}0.11 \pm \\
0.092\end{array}$ & $\begin{array}{c}0.06 \pm \\
0.08 \\
45.45\end{array}$ & $\begin{array}{c}0.049 \pm \\
0.071 \\
(55.45)\end{array}$ & $\begin{array}{c}0.03 \pm \\
0.071 \\
(72.72)\end{array}$ & $\begin{array}{c}0.02 \pm \\
0.05 \\
(81.81)\end{array}$ & $\begin{array}{c}0.015 \pm \\
0.03 \\
(85.63)\end{array}$ & $\begin{array}{c}0.0125 \pm \\
0.022 \\
(88.63)\end{array}$ & $\begin{array}{c}0.011 \pm \\
0.02 \\
(90)\end{array}$ \\
\hline IQVI 4 & $\begin{array}{l}0.11 \pm \\
0.052\end{array}$ & $\begin{array}{c}0.09 \pm \\
0.044 \\
(18.18)\end{array}$ & $\begin{array}{c}0.08 \pm \\
0.04 \\
(27.27)\end{array}$ & $\begin{array}{c}0.071 \pm \\
0.017 \\
(35.45)\end{array}$ & $\begin{array}{c}0.062 \pm \\
0.013 \\
(43.63)\end{array}$ & $\begin{array}{c}0.06 \pm \\
0.011 \\
(45.45)\end{array}$ & $\begin{array}{c}0.05 \pm \\
0.01 \frac{1}{2} \\
(54.54)\end{array}$ & $\begin{array}{c}0.05+ \\
0.007 \\
(54.54)\end{array}$ \\
\hline IQVI 5 & $\begin{array}{c}0.101 \pm \underline{+} \\
0.112\end{array}$ & $\begin{array}{c}0.05 \pm \\
0.11 \\
(50.49)\end{array}$ & $\begin{array}{c}0.05 \pm \\
0.11 \\
(50.49)\end{array}$ & $\begin{array}{c}0.046 \pm \\
0.07 \\
(54.45)\end{array}$ & $\begin{array}{c}0.037 \pm \\
0.06 \\
(63.26)\end{array}$ & $\begin{array}{c}0.032 \pm \\
0.04 \\
(68.31)\end{array}$ & $\begin{array}{c}0.021 \pm \\
0.03 \\
(79.20)\end{array}$ & $\begin{array}{c}0.02 \pm \\
0.02 \\
(80.19)\end{array}$ \\
\hline IQVI 6 & $\begin{array}{l}0.14 \underline{+} \\
0.019\end{array}$ & $\begin{array}{c}0.102 \pm \\
0.04 \overline{4} \\
(27.12)\end{array}$ & $\begin{array}{c}0.099 \pm \\
0.01 \\
(29.28)\end{array}$ & $\begin{array}{c}0.09 \pm \\
0.007 \\
(37.71)\end{array}$ & $\begin{array}{c}0.09 \pm \\
0.007 \\
(37.71)\end{array}$ & $\begin{array}{c}0.06+ \\
0.0069 \\
(57.14)\end{array}$ & $\begin{array}{c}0.05 \pm \\
0.005 \\
(64.28)\end{array}$ & $\begin{array}{c}0.05 \pm \\
0.005 \\
(64.28)\end{array}$ \\
\hline IQVI 7 & $\begin{array}{c}0.13+ \\
0.0192\end{array}$ & $\begin{array}{c}0.12+ \\
0.018 \\
(7.6)\end{array}$ & $\begin{array}{c}0.112+ \\
0.016 \\
(13.84)\end{array}$ & $\begin{array}{c}0.10+ \\
0.013 \\
(23.07)\end{array}$ & $\begin{array}{l}0.09+ \\
0.003 \\
30.76\end{array}$ & $\begin{array}{c}0.08+ \\
0.002 \\
(38.46)\end{array}$ & $\begin{array}{l}0.07 \pm \\
0.001 \\
46.15\end{array}$ & $\begin{array}{l}0.06+ \\
0.001 \\
53.84\end{array}$ \\
\hline IQVI8 & $\begin{array}{l}0.101 \pm \\
0.0162\end{array}$ & $\begin{array}{c}0.102 \pm \\
0.044 \\
(27.12)\end{array}$ & $\begin{array}{c}0.059 \pm \\
0.012 \\
(41.58)\end{array}$ & $\begin{array}{c}0.04 \pm \\
0.01 \\
(60.39)\end{array}$ & $\begin{array}{l}0.028 \pm \\
0.0091 \\
(72.27)\end{array}$ & $\begin{array}{l}0.02 \pm \\
0.0091 \\
(80.19)\end{array}$ & $\begin{array}{c}0.01 \pm \\
0.006 \\
(90.09)\end{array}$ & $\begin{array}{c}0.009 \pm \\
0.004 \\
(91.08)\end{array}$ \\
\hline IQVI 9 & $\begin{array}{c}0.13 \pm \\
0.0192\end{array}$ & $\begin{array}{c}0.12 \pm \\
0.018 \\
(7.6)\end{array}$ & $\begin{array}{c}0.112 \pm \\
0.016 \\
(13.84)\end{array}$ & $\begin{array}{c}0.10 \pm \\
0.015 \\
(23.07)\end{array}$ & $\begin{array}{c}0.09 \pm \\
0.01 \\
(30.76)\end{array}$ & $\begin{array}{c}0.08 \pm \pm \\
0.009 \\
(38.46)\end{array}$ & $\begin{array}{c}0.08 \pm \\
0.009 \\
(38.46)\end{array}$ & $\begin{array}{c}0.07 \pm \\
0.002 \\
(59.40)\end{array}$ \\
\hline & $\begin{array}{l}0.11 \pm t \\
0.016\end{array}$ & $\begin{array}{l}0.09 \pm \\
0.015 \\
18.18\end{array}$ & $\begin{array}{c}0.08 \pm \\
0.016 \\
(27.27)\end{array}$ & $\begin{array}{c}0.05 \pm \\
0.006 \\
(54.54)\end{array}$ & $\begin{array}{c}0.03 \pm \\
0.005 \\
(72.72)\end{array}$ & $\begin{array}{c}0.02 \pm \\
0.004 \\
(81.81)\end{array}$ & $\begin{array}{c}0.019 \pm \\
0.003 \\
(82.72)\end{array}$ & $\begin{array}{c}0.011 \pm \\
0.002 \\
(90)\end{array}$ \\
\hline IQVI 11 & $\begin{array}{c}0.13 \pm \\
0.0192\end{array}$ & $\begin{array}{c}0.122 \pm \\
0.018 \\
(6.15)\end{array}$ & $\begin{array}{l}0.088+ \\
0.0127 \\
(38.46)\end{array}$ & $\begin{array}{c}0.08 \pm \\
0.012 \\
(38.46)\end{array}$ & $\begin{array}{c}0.07 \pm \\
0.09 \\
(46.15)\end{array}$ & $\begin{array}{c}0.04 \pm \\
0.03 \\
(69.23)\end{array}$ & $\begin{array}{c}0.03 \pm \\
0.01 \\
(76.92)\end{array}$ & $\begin{array}{c}0.02 \pm \\
0.009 \\
(84.61)\end{array}$ \\
\hline IQVI 12 & $\begin{array}{c}0.101+ \\
0.192\end{array}$ & $\begin{array}{l}0.089 \\
\pm 0.17 \\
11.88\end{array}$ & $\begin{array}{c}0.069 \pm \\
0.012 \\
(31.68)\end{array}$ & $\begin{array}{c}0.06+ \\
0.098 \\
(40.59)\end{array}$ & $\begin{array}{c}0.06 \pm \\
0.09 \\
(40.59)\end{array}$ & $\begin{array}{c}0.041 \pm \\
0.05 \\
(59.4)\end{array}$ & $\begin{array}{c}0.037 \pm \\
0.03 \\
(63.36)\end{array}$ & $\begin{array}{c}0.01+ \\
0.027 \\
(90.09)\end{array}$ \\
\hline IQVI 13 & $\begin{array}{l}0.101+ \\
0.0192\end{array}$ & $\begin{array}{c}0.06+ \\
0.017 \\
(40.59)\end{array}$ & $\begin{array}{c}0.059 \\
+0.016 \\
(41.58)\end{array}$ & $\begin{array}{c}0.04+ \\
0.01 \overline{51} \\
(60.39)\end{array}$ & $\begin{array}{c}0.03+ \\
0.008 \\
(70.29)\end{array}$ & $\begin{array}{c}0.0162 \pm \\
0.009 \\
(83.96)\end{array}$ & $\begin{array}{l}0.0153 \\
0.007 \pm \\
(84.85)\end{array}$ & $\begin{array}{c}0.0074 \underline{+} \\
0.007 \\
(92.67)\end{array}$ \\
\hline IQVI14 & $\begin{array}{l}0.12+ \\
0.019\end{array}$ & $\begin{array}{c}0.05 \pm \\
0.019 \\
(58.33)\end{array}$ & $\begin{array}{c}0.048 \pm \\
0.009 \\
(60)\end{array}$ & $\begin{array}{c}0.04+ \\
0.007 \\
(66.66)\end{array}$ & $\begin{array}{c}0.035 \pm \\
0.006 \\
(70.83)\end{array}$ & $\begin{array}{c}0.03+ \\
0.003 \\
(75)\end{array}$ & $\begin{array}{c}0.01 \pm \\
0.001 \\
(91.66)\end{array}$ & $\begin{array}{c}0.009 \pm \\
0.001 \\
(92.56)\end{array}$ \\
\hline IQVI 15 & $\begin{array}{c}0.101+ \\
0.192\end{array}$ & $\begin{array}{c}0.041+ \\
0.137 \\
(59.40) \\
\end{array}$ & $\begin{array}{c}0.04+ \\
0.016 \\
(60.39) \\
\end{array}$ & $\begin{array}{c}0.038 \pm \\
0.01 \\
(62.37) \\
\end{array}$ & $\begin{array}{c}0.03 \pm \\
0.01 \\
(70.29) \\
\end{array}$ & $\begin{array}{c}0.02+\frac{+}{3} \\
0.003 \\
(80.19) \\
\end{array}$ & $\begin{array}{c}0.01 \pm \\
0.003 \\
(90.09) \\
\end{array}$ & $\begin{array}{c}0.008 \pm \\
0.002 \\
(92.07) \\
\end{array}$ \\
\hline
\end{tabular}

One-way ANOVA followed by Dunnett's post HOC test; The numbers given in the brackets were the \% scavenging. 


\section{Anti-inflammatory activity}

\section{Carrageenan induced rat hind paw edema method}

The method of Winter et al. ${ }^{12}$ was used with slight modification. The apparatus used for the measurement of rat paw volume was phlethismograph. The animals were divided into eight groups of six animals each. One group served as a standard (Ibuprofen) and another group served as control (1\% CMC) and rest of the groups were used for the test drugs. Food was withdrawn overnight with adequate water before the experiment. The drugs were given orally. After $1 \mathrm{~h}$, a sub plantar injection of $0.05 \mathrm{~mL}$ of $1 \%$ carrageenan was administered. The volume of the injected paw was measured with a plethysmograph immediately. The paw volume was again measured after $3 \mathrm{~h}$. The average paw volume in a group of drug treated rats was compared with that of a group with vehicle (control group) and the percentage inhibition of oedema was calculated using the formula (Table 4).

$$
\% \text { Inhibition }=(1-\mathrm{Vt} / \mathrm{Vc}) \times 100
$$

$\mathrm{Vt}=$ Mean volume of the test drug, $\mathrm{Vc}=$ Mean volume of the control.

Table 4. Anti-inflammatory activity of phenyl pyrazolo indoloquinoxaline derivatives

\begin{tabular}{ccccc}
\hline S.No. & Compound & Dose, $\mathrm{mg} / \mathrm{kg}$ & $\begin{array}{c}\text { Mean edema volume } \\
\pm \mathrm{S} . \mathrm{E}, .0-3 \mathrm{~h}\end{array}$ & \% Reduction \\
\hline 1. & Control & & $0.40 \pm 0.162$ & \\
2. & Ibuprofen & 200 & $0.03 \pm 0.15$ & 92.5 \\
3. & IQVI3 & 200 & $0.24 \pm 0.145^{\mathrm{a}}$ & 40.0 \\
4. & IQVI5 & 200 & $0.26 \pm 0.14^{\mathrm{a}}$ & 35.0 \\
5. & IQVI8 & 200 & $0.25 \pm 0.11^{\mathrm{a}}$ & 37.5 \\
6. & IQVI13 & 200 & $0.09 \pm 0.02^{\mathrm{a}}$ & 77.5 \\
7 & IQVI14 & 200 & $0.075 \pm 0.003^{\mathrm{a}}$ & 81.2 \\
8. & IQVI15 & 200 & $0.04 \pm 0.0014^{\mathrm{a}}$ & 90.0 \\
\hline
\end{tabular}

One-way ANOVA followed by Schiff's post HOC test. Allowance value $=0.239 ; a=P<0.05$ (Vs) control.

Anti-histaminic activity ${ }^{13}$

Histamine chamber method

In this method thirty two healthy adult guinea pigs of either sex divided into 8 group of 2 animals each weighing around $400 \mathrm{~g}$, fasted overnight, were kept in histamine chamber and exposed to histamine aerosol $(0.5 \%$ aqueous solution of histamine acid phosphate in a Nebulizer) until they collapse. Those that collapse within 2 minutes were revived with fresh air and used for this test. Twelve hours later, the animals were given an oral dose of test compound suspended in $1 \%$ acacia solution and after $1 \mathrm{~h}$ for absorption, the guinea pigs were again exposed to the same concentration of histamine aerosol. Those that do not collapse within 6 minutes are deemed protected. Percentage protection has been measured by calculating the time of onset of convulsions (Table 5).

\section{Results and Discussion}

All the compounds were synthesized through the depicted in the Scheme 1 and confirmed by IR, ${ }^{1} \mathrm{H}$ NMR, mass spectroscopy. Indoquinaxoline and diphenyl quinaxoline and both these quinoxalines were connected with methylene bridge, which is then allowed for acetylation. The acetylated product (IQ IV) was made to react with different aromatic aldehydes to give chalcones (IQV 1-IQV 5). Chalcones refluxed with substituted acid hydrazides to afford different phenylpyrazolo indoloquinoxaline derivatives (IQVI 1-IQVI 15). All the compounds shown significant antioxidant activity among them IQVI-3 45.45\%, IQVI 5 50.9\%, IQVI 8 27.12\%, IQVI13 40.59\%, IQVI $1458.33 \%$ and IQVI $1559.40 \%$ showed good free radical scavenging activity (Table 3). In the anti-inflammatory activity, compounds IQVI $\mathbf{1 3}$ 77.50\% IQVI 1481.25 and IQVI 15 90.00\% showed good inhibition of edema volume (Table 4) 
and compounds IQVI $390.4 \%$, IQVI 4 90.7\% IQVI $890.5 \%$ and IQVI $1090.9 \%$ showed good $\%$ protection of antihistamic activity (Table 5).

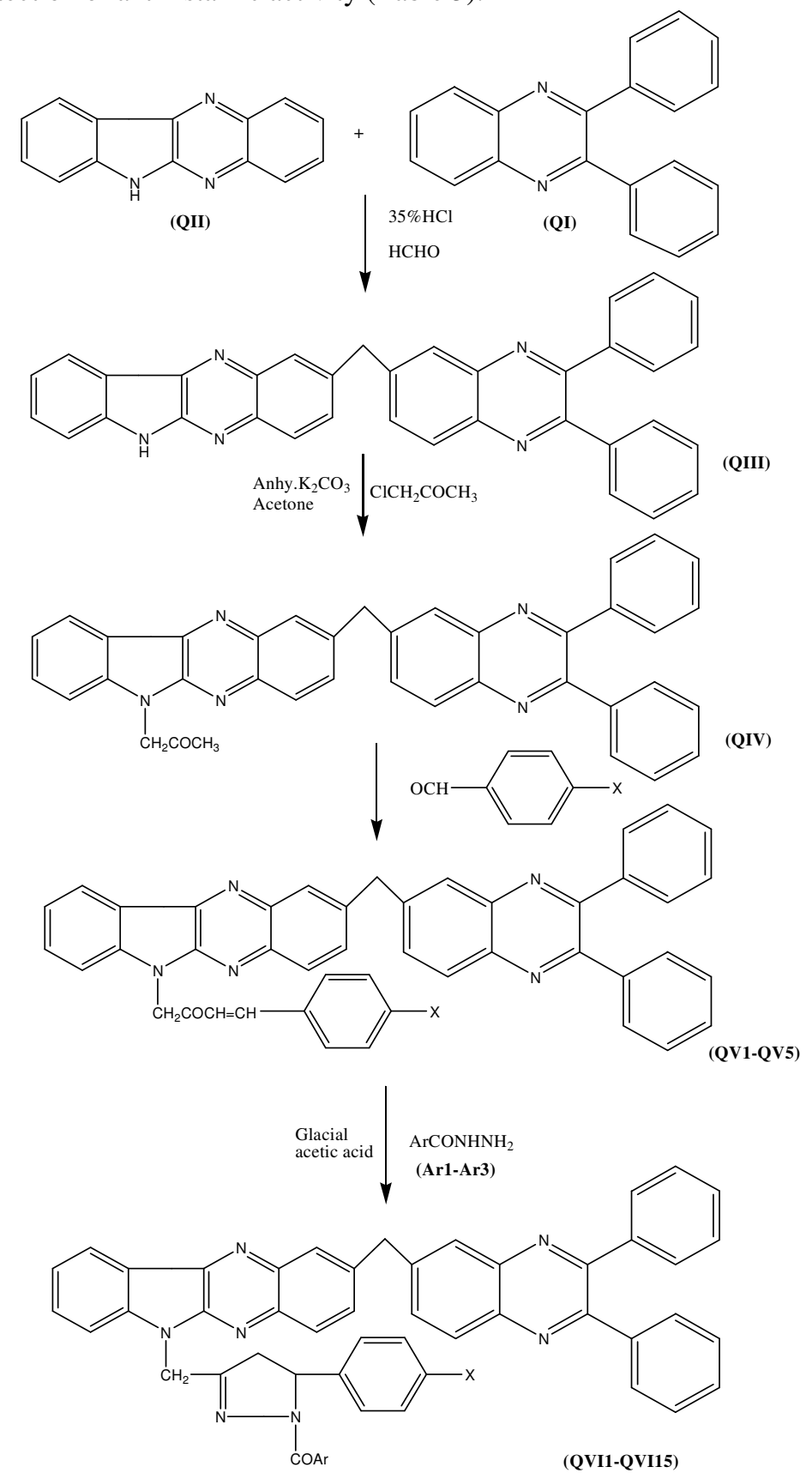

$\mathrm{X}=\mathrm{H}, \mathrm{OH}, \mathrm{Cl}, \mathrm{F}, \mathrm{OCH}_{3}$

$\mathrm{Ar}=-\mathrm{C}_{6} \mathrm{H}_{5},-\mathrm{C}_{6} \mathrm{H}_{4} \mathrm{OH},-\mathrm{C}_{6} \mathrm{H}_{4} \mathrm{Cl}$

Scheme 1 
Table 5. Antihistaminic activity of phenylpyrazolo indoloquinoxaline derivatives

\begin{tabular}{cccccc}
\hline Comp & $\mathrm{X}$ & $\mathrm{Ar}$ & Mol.formula & $\begin{array}{c}\text { Onset of Convulsions } \\
\text { (s) Mean } \pm \text { SD }\end{array}$ & $\begin{array}{c}\% \\
\text { Protection }\end{array}$ \\
\hline IQVI 1 & $\mathrm{H}$ & $\mathrm{C}_{6} \mathrm{H}_{5}$ & $\mathrm{C}_{52} \mathrm{H}_{37} \mathrm{~N}_{7} \mathrm{O}$ & $994 \pm 90$ & 89.1 \\
IQVI 2 & $\mathrm{OH}$ & $\mathrm{C}_{6} \mathrm{H}_{5}$ & $\mathrm{C}_{52} \mathrm{H}_{37} \mathrm{~N}_{7} \mathrm{O}_{2}$ & $1045 \pm 98$ & 89.6 \\
IQVI 3 & $\mathrm{F}$ & $\mathrm{C}_{6} \mathrm{H}_{5}$ & $\mathrm{C}_{52} \mathrm{H}_{36} \mathrm{~N}_{7} \mathrm{O}_{3} \mathrm{~F}$ & $1125 \pm 92$ & 90.4 \\
IQVI 4 & $\mathrm{Cl}$ & $\mathrm{C}_{6} \mathrm{H}_{5}$ & $\mathrm{C}_{52} \mathrm{H}_{36} \mathrm{~N}_{7} \mathrm{OCl}$ & $1165 \pm 96$ & 90.7 \\
IQVI 5 & $\mathrm{OCH}_{3}$ & $\mathrm{C}_{6} \mathrm{H}_{5}$ & $\mathrm{C}_{53} \mathrm{H}_{39} \mathrm{~N}_{7} \mathrm{O}_{2}$ & $945 \pm 91$ & 88.5 \\
IQVI 6 & $\mathrm{H}$ & $\mathrm{OHC}_{6} \mathrm{H}_{4}$ & $\mathrm{C}_{52} \mathrm{H}_{37} \mathrm{~N}_{7} \mathrm{O}_{2}$ & $964 \pm 90$ & 89.7 \\
IQVI 7 & $\mathrm{OH}$ & $\mathrm{OHC}_{6} \mathrm{H}_{4}$ & $\mathrm{C}_{52} \mathrm{H}_{37} \mathrm{~N}_{7} \mathrm{O}_{3}$ & $1074 \pm 93$ & 89.9 \\
IQVI 8 & $\mathrm{F}$ & $\mathrm{OHC}_{6} \mathrm{H}_{4}$ & $\mathrm{C}_{52} \mathrm{H}_{36} \mathrm{~N}_{7} \mathrm{O}_{2} \mathrm{~F}$ & $1137 \pm 94$ & 90.5 \\
IQVI 9 & $\mathrm{Cl}$ & $\mathrm{OHC}_{6} \mathrm{H}_{4}$ & $\mathrm{C}_{52} \mathrm{H}_{36} \mathrm{~N}_{7} \mathrm{O}_{2} \mathrm{Cl}$ & $1024 \pm 95$ & 89.4 \\
IQVI 10 & $\mathrm{OCH}$ & $\mathrm{OHC}_{6} \mathrm{H}_{4}$ & $\mathrm{C}_{53} \mathrm{H}_{39} \mathrm{~N}_{7} \mathrm{O}_{3}$ & $1194 \pm 96$ & 90.9 \\
IQVI 11 & $\mathrm{H}$ & $\mathrm{ClC}_{6} \mathrm{H}_{4}$ & $\mathrm{C}_{52} \mathrm{H}_{36} \mathrm{~N}_{7} \mathrm{O} \mathrm{Cl}$ & $980 \pm 92$ & 88.9 \\
IQVI 12 & $\mathrm{OH}$ & $\mathrm{ClC}_{6} \mathrm{H}_{4}$ & $\mathrm{C}_{52} \mathrm{H}_{36} \mathrm{~N}_{7} \mathrm{O}_{2} \mathrm{Cl}$ & $999 \pm 95$ & 89.1 \\
IQVI 13 & $\mathrm{F}$ & $\mathrm{ClC}_{6} \mathrm{H}_{4}$ & $\mathrm{C}_{52} \mathrm{H}_{35} \mathrm{~N}_{7} \mathrm{OClF}$ & $828 \pm 86$ & 86.96 \\
IQVI 14 & $\mathrm{Cl}$ & $\mathrm{ClC}_{6} \mathrm{H}_{4}$ & $\mathrm{C}_{52} \mathrm{H}_{35} \mathrm{~N}_{7} \mathrm{OCl}$ & $1012 \pm 94$ & 89.33 \\
IQVI 15 & $\mathrm{OCH}$ & $\mathrm{ClC}_{6} \mathrm{H}_{4}$ & $\mathrm{C}_{53} \mathrm{H}_{38} \mathrm{~N}_{7} \mathrm{O}_{2} \mathrm{Cl}$ & $756 \pm 82$ & 85.71 \\
Control & & & & $108 \pm 12$ & \\
CPM & & & & $1228 \pm 65$ & 91.20 \\
\hline
\end{tabular}

\section{Conclusion}

Indoquinoxaline and diphenylquinoxaline both these quinoxalines were connected with methylene bridge, which is then allowed for acetylation. The acetylated product (IQ IV) is made to react with different aromatic aldehydes to give chalcones (IQV 1-IQV 5). Chalcones refluxed with substituted acid hydrazides to afford different phenyl pyrazolo indoloquinoxaline derivatives (IQVI 1-IQVI 15). The synthesized compounds were checked for antioxidant, anti-inflammatory and antihistamic activity.

\section{Acknowledgment}

The authors are thankful to IICT Hyderabad, spectral analysis. Also thankful to Geethanjali College of Pharmacy for providing facilities to carry out this research work.

\section{References}

1. Sandeep Kotharkar A and Devender shinda B, Bio Org Med Chem Lett., 2006, 16, 6181.

2. Dubey P K, Naidu A, Vijaya S and George B Vineel, Indian J Chem., 2005, 44B, 573.

3. Ganapathy S, Ramalingam P and Babu Rao Ch, Indian J Heterocycl Chem., 2007, 16, 283.

4. Kumar A, Sharma S and Bajaj K, Indian J Chem., 2003, 42B(8), 1979.

5. Ragabasawaraj Bodkey Yadav and Sangapure S S, Indian J Heterocycl Chem., 2001,11, 31.

6. Heas V, Roelof, Grosseurt and Cornelis A, European Pat Appl., 1981, 21, 506.

7. Suthakaran R, Nagarajan G, Balasubramaniam V, Suganthi K and Velrajan G, Indian J Heterocycl Chem., 2005, 14, 201.

8. Leonard J T, Yagnapriya S, Sridhar S K and Gunasekaran V, Indian J Heterocycl Chem., 2005, 14, 377.

9. Suthakaran.R, Somasekhar G, Sridevi Ch, Mari kannan M, Suganthi K and Nagarajan G, Asian J Chem., 2007, 5, 3353.

10. Harinadha babu V, Sridevi Ch, Joseph A and Srinivasan K K, Indian J Pharm Sci., 2007, 66(8), 470.

11. Sreejayan N and Rao M N, Int J Pharm., 1993, 100, 93-97.

12. Winter C A, Risley E A and Nuss G W, Proc Soc Exp Biol Med., 1962, 111, 544-547.

13. Bhargava P N and Chaurasia M R, J Med Chem., 1968, 11(4), 908-909. 


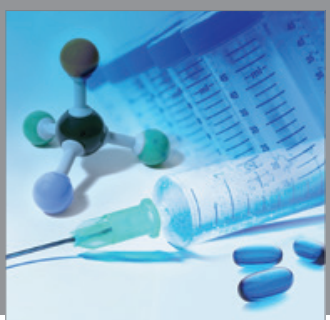

International Journal of

Medicinal Chemistry

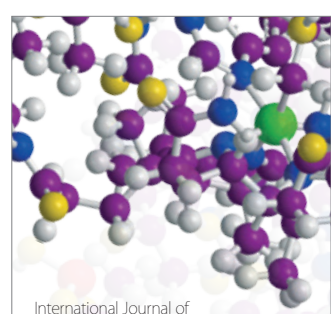

Carbohydrate Chemistry

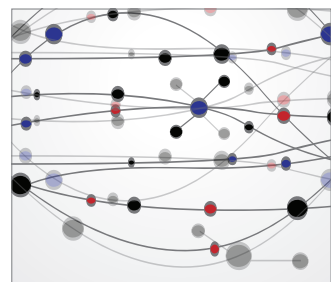

The Scientific World Journal
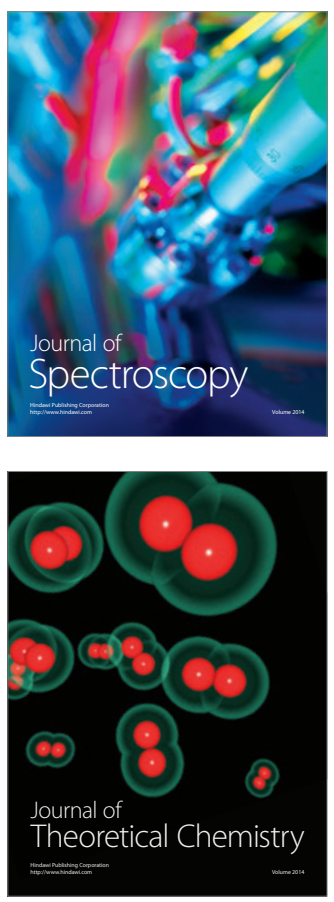
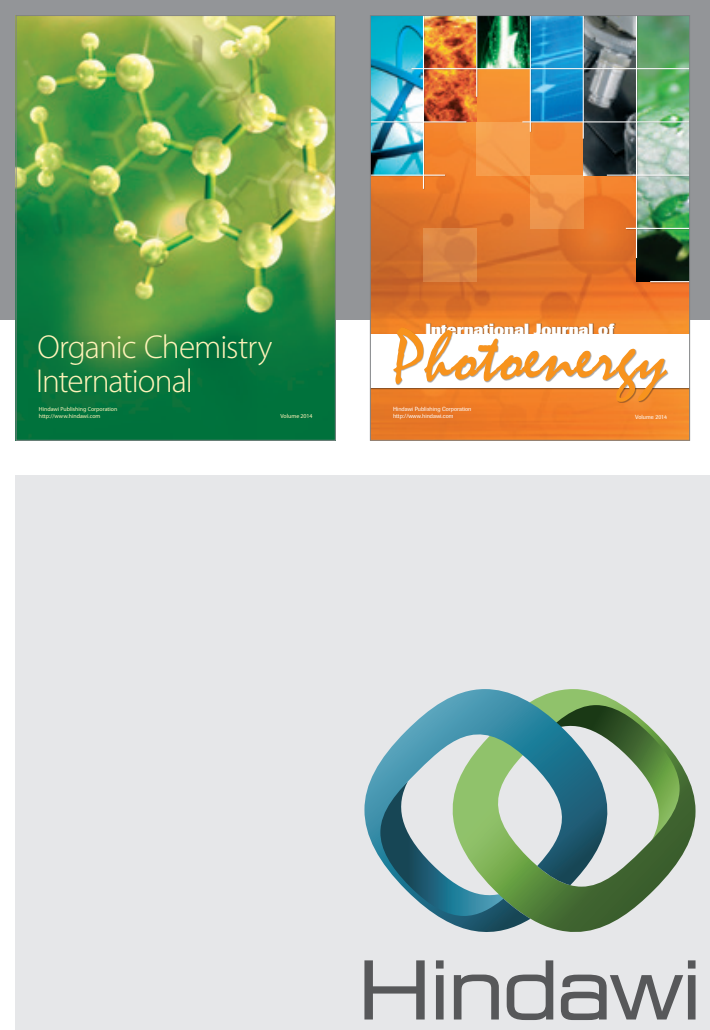

Submit your manuscripts at

http://www.hindawi.com
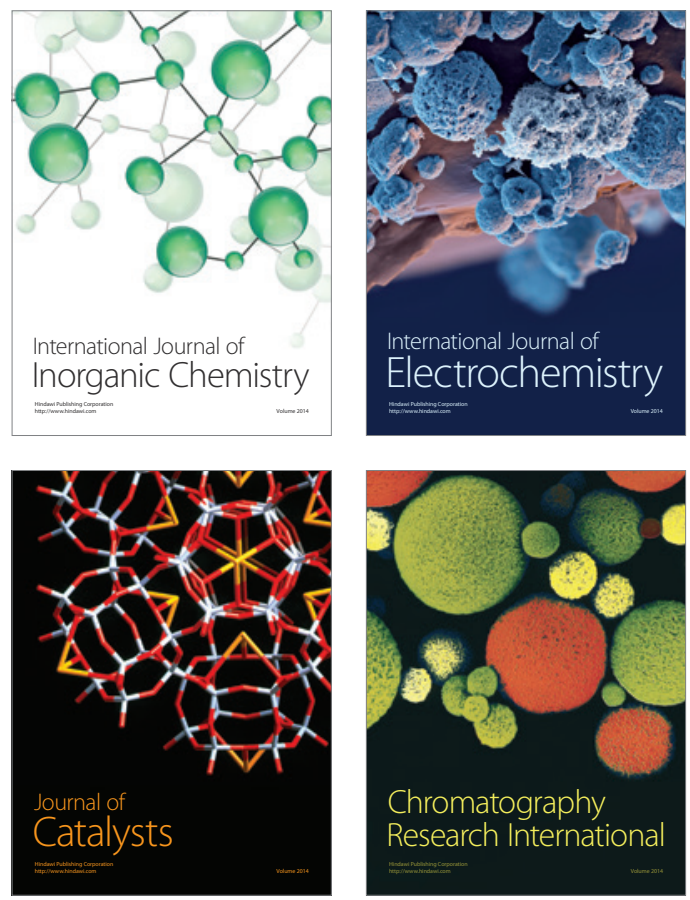
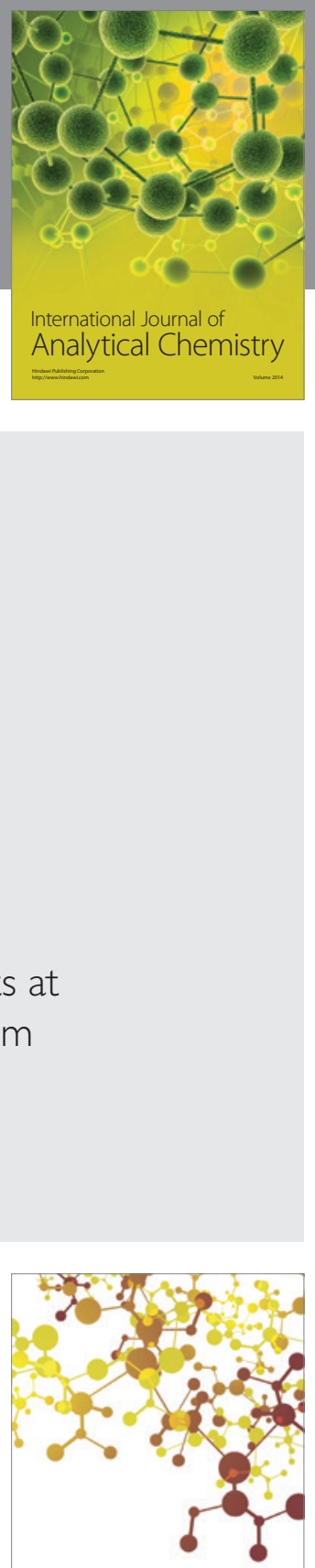

Journal of

Applied Chemistry
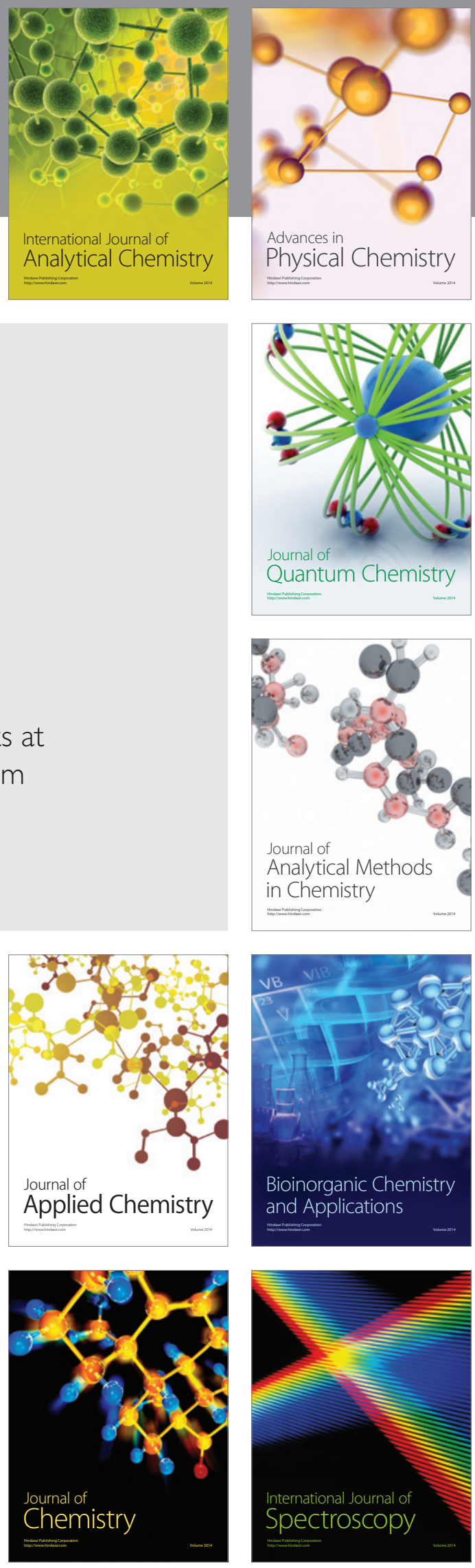